\title{
Precipitating Factors, Clinical Features and Outcome of Diabetic Ketoacidosis in Children in a Tertiary Care Hospital of Bangladesh
}

\author{
Yasmin $\mathrm{F}^{\mathrm{a}^{*}}$, Ahmed MAU ${ }^{*}$, Morshed ASM ${ }^{\mathrm{c}}$, Begum $\mathrm{T}^{\mathrm{d}}$, Mohsin Fe $\mathrm{F}^{\mathrm{e}}$ Baki MA
}

\begin{abstract}
Background: Diabetic ketoacidosis (DKA) is a major complication of type 1 and type 2 diabetes mellitus and is associated with increased risk of morbidity and mortality. Infections, non-compliance and co-morbid states are most important precipitating causes. Proper identification of the precipitating factor is very important in management of DKA. Clinical feature and management of DKA are well known and have been described in many text books and reviews in literature. However, there are a very few published large studies from Bangladesh. For this reason, this study had evaluated fifty children with Diabetic ketoacidosis and to identify their clinical features, precipitating factors and outcome.
\end{abstract}

Methods: This observational study was done among admitted children with DKA in the deptartment of Paediatrics of Bangladesh Institute of Research and Rehabilitation for Diabetes, Endocrine and Metabolic Disorders (BIRDEM) General Hospital during study period between September 2016 to February 2017.

Results: Fifty children were admitted with Diabetic ketoacidosis. Seventy percent were new diabetes cases and the remaining (30\%) were known diabetic patient. Majority (62\%) were female. Mean age was 9.31 years with 4.40 standard deviation. Infection was the commonest (62\%) precipitating factor followed by insulin omission (10\%). Major clinical features were dehydration (100\%), polyuria (98\%), Kussmaul's breathing (60\%) and abdominal pain (38\%). Eighty four percent patients improved after treatment and $16 \%$ patient developed complications like acute kidney injury and septicaemia. There was no mortality.

Conclusion: Infection was the commonest precipitating factor of DKA. Kussmaul's breathing and dehydration were the commonest clinical features. Most of the patients improved after treatment.

Key words: Bangladesh, children, clinical presentation, diabetic ketoacidosis, outcome, precipitating factors.

(BIRDEM Med J 2019; 9(2): 121-126)

\section{Author Information}

a. Dr. Farzana Yasmin, Resident medical officer, Dept. of Paediatrics, BIRDEM General Hospital.

b. Dr. Md. Ashraf Uddin Ahmed, Resident physician, BIRDEM General Hospital.

c. Dr. A.S.M. Morshed, Asst. Professor, Dept. of psychiatry Sirajul Islam Medical College.

d. Dr. Tahmina Begum, Professor of Paediatrics

e. Dr. Fouzia Mohsin, Professor, Dept. of Paediatrics, BIRDEM General Hospital

f. Dr. M.A. Baki, Assistant Professor, Dept. of Paediatrics, BIRDEM General Hospital

*First two authors had equal contributions and will be considered as first authors.

Address of Correspondence: Dr. Md. Ashraf Uddin Ahmed, Resident physician, Room no 121, Outpatient department, ground floor, BIRDEM General Hospital, 122, Kazi Nazrul Islam Avenue, Sahbagh, Dhaka-1000. Email: shakilbm14@yahoo.com.

Received: June 5, 2018

Accepted: February 28, 2019

\section{Introduction}

Diabetes mellitus (DM) is one of the main threat to human health in the $21^{\text {st }}$ century. Recently compiled data shows that approximately 150 million people have DM worldwide and that this number will be double by the year $2025 .^{1}$

If left untreated, diabetes can cause many complications. Acute complications include diabetic ketoacidosis (DKA) and hyperosmolar hyperglycemic state. $^{2}$

DKA is an acute complication and medical emergency in children and adolescent with diabetes. It may be the presenting feature of type 1 diabetes, but more frequently it occurs in established diabetes patients- both type 1 and type $2 .^{2}$ It is characterized by a biochemical 
triad of hyperglycemia, ketonemia (ketonuria) and acidosis. It is caused by a decrease in effective circulating insulin associated with elevation in counterregulatory hormones. ${ }^{3,4}$ It is the most frequent cause of diabetes-related death in children with the mortality rate ranging between $6 \%$ and $24 \%$ in developing countries. ${ }^{5}$ Studies have documented a $2 \%$ to $24 \%$ mortality rate in patients who develop cerebral edema with DKA, hence accounting for most of the DKA deaths and a high rate of permanent neurologic morbidity. ${ }^{1,6}$ Other causes of morbidity and mortality in DKA include hypoglycaemia, infections, pulmonary edema, central nervous system hemorrhage or thrombosis, other large vessel thrombosis, cardiac arrhythmias caused by electrolyte disturbances, pancreatitis, acute renal failure and intestinal necrosis. $^{5}$

In Bangladesh, no epidemiological study on childhood DKA has been done so far. In a one-year review, the proportion of diabetics under 18 years of age was found to be $1.34 \%$ among the registered cases of BIRDEM General Hospital. ${ }^{7}$

A very few studies have evaluated data describing clinical profile, precipitating factors and outcomes of children with diabetic ketoacidosis. This study was done among fifty children with diabetic ketoacidosis to identify their clinical features, precipitating factors and outcome.

\section{Methods}

This was an observational study, carried out in the Department of Paediatrics, BIRDEM General Hospital, Dhaka from September 2016 to February 2017.

All admitted children with DKA in the department of Paediatrics, BIRDEM General Hospital during study period with a diagnosis of DKA, whether previously known diabetic or newly diagnosed case were included in the study while patients having other causes of acidosis like chronic kidney disease and diarrhea were excluded.

After applying the selection criteria, a total of 50 patients including 31 females and 19 males participated in this study. Each parent of patient enrolled in the survey, conducted in full accordance with the World Medical
Association Declaration of Helsinki, was given a detailed verbal and written description of the proposed study in their own language and signed consent for participation was obtained. Ethical approval for the study was obtained from ethical review committee. A typed questionnaire, in English and Bengali, was given to all the patients. The participants were assured that the information acquired will be used for academic purpose only.

Data were analyzed by computer with the help of Statistical Package for Social Sciences (SPSS) version 20.0. Statistical analyses were done by using appropriate statistical tool like 'Chi-square' test, student's 't' test, where applicable. Statistical significance was set at $<0.05$ level and confidence interval at 95\% level.

\section{Results}

Total number of patients were 50, male were 19 (38\%) and female were $31(62 \%)$. Mean age of the study population was $9.31 \pm 4.4$ years. Among the 50 patients, $15(30 \%)$ were known diabetic patients. Base-line characteristics and status of DM are presented in Table I and Table II respectively. DKA occurred among patients with poor glycaemic control (HbAlc $>7 \%$ in $100 \%$.

Table I Base-line characteristics of the study population $(\mathrm{N}=50)$

\begin{tabular}{lc} 
Characteristics & Results \\
\hline Mean age (years) & $9.31 \pm 4.4$ \\
Male: Female & $1: 1.6$ \\
Known DM: New diagnosis of DM & $1: 2.33$ \\
Rural: Urban & $1: 1.28$ \\
Mean RBG* at admission (mmol/L) & 26.13 \\
Mean HbAlc (\%) & 12.25 \\
\hline
\end{tabular}

$* \mathrm{RBG}=$ random blood glucose

Severe DKA $(\mathrm{pH}<7)$ was less common (Table III). Common presentations and precipitating causes are shown in Table IV and Table V respectively. 
Table II Status of DM in the study population $(\mathrm{N}=50)$

\begin{tabular}{lcc} 
Status of DM & Frequency & Percentage \\
\hline Newly detected DM & 35 & 70 \\
Treatment of cases $(\mathrm{n}=15)$ & & \\
Insulin & 14 & 28 \\
OAD* & 1 & 2 \\
RBG at presentation (m.mol/L) & & \\
$<20$ & 5 & 10 \\
$21-25$ & 17 & 34 \\
$26-30$ & 21 & 42 \\
$31-35$ & 7 & 14 \\
HbAlc $(\%)$ & & \\
$<7$ & 0 & 0 \\
$7-8.5$ & 6 & 12 \\
$8.6-10$ & 4 & 8 \\
$>10$ & 40 & 80 \\
\hline
\end{tabular}

*OAD $=$ oral anti-diabetic drugs,

Table III Severity of DKA3 of the study population $(\mathrm{N}=50)$

\begin{tabular}{lcc}
$\mathrm{pH}$ & Frequency & Percentage \\
\hline$<7$ & 18 & 36 \\
$7-7.24$ & 16 & 32 \\
$7.25-7.30$ & 16 & 32 \\
\hline
\end{tabular}

Table IV Clinical presentations of the study populations $(\mathrm{N}=50)$

\begin{tabular}{lcc} 
Presenting complaints & Frequency & Percentage \\
\hline Polyuria & 49 & 98.0 \\
Weight loss & 26 & 52.0 \\
Abdominal pain & 19 & 38.0 \\
Respiratory distress & 27 & 54.0 \\
Confusion & 1 & 2.0 \\
Blurring vision & 1 & 2.0 \\
Polydipsia & 41 & 82.0 \\
Vomiting & 27 & 54.0 \\
Fever & 17 & 34.0 \\
Drowsiness & 20 & 40.0 \\
Coma & 2 & 4.0 \\
Weakness & 30 & 60.0 \\
\hline
\end{tabular}

Table V Precipitating causes of DKA $(\mathrm{N}=50)$

\begin{tabular}{lcc} 
Precipitating Causes & Frequency & Percentage \\
\hline Infection & 31 & 62.0 \\
Omission of insulin & 5 & 10.0 \\
Reduction of insulin & 1 & 2.0 \\
Unidentified & 13 & 26.0 \\
\hline Total & 50 & 100.0 \\
\hline
\end{tabular}

Most of the patient (54\%) had hyponatraemia at presentation while $58 \%$ patients required potassium infusion during treatment. Eight $(16 \%)$ patients were complicated with acute kidney injury. Most of the patients $(42 \%)$ suffered from urinary tract infection followed by pneumonia ( $8 \%$ ) and skin infection $(2 \%)$. There was no mortality.

\section{Discussion}

Our data shows no association between age and severity of DKA. These findings are also consistent with the results of a study in which although age was found to be significantly associated with the incidence of DKA, but not with its severities. ${ }^{8}$ Among the study population $35(70 \%)$ patients were newly diagnosed diabetic and $15(30 \%)$ patients were already diagnosed as diabetic.

Approximately three-quarter (70\%) of the children and adolescents in the present study manifested with DKA at first diagnosis of diabetes. This is not surprising as a similar high prevalence rate has been reported in a previous study in Nigeria. ${ }^{9}$ In contrast, a study in Northern Finland reported a significant decline in frequency (18.9 versus $29.5 \%$ ) of DKA at first presentation of type $1 \mathrm{DM}$ over the later 10 years of a 20 -year review. ${ }^{10}$ The explanation is that the high background prevalence of diabetes mellitus in Finland makes the recognition of the disease easier by both the public and the physician. Increased level of awareness of early symptoms of diabetes among the population and greater medical alertness to the occurrence of type IDM among the physicians will be rewarding.

Consistent with previous report ${ }^{11}$, there was a female preponderance $(62 \%)$ among DKA patients in the present study. This finding may be explained by the hormonal changes which accompany puberty, 
particularly the elevation in the serum levels of some counter regulatory hormones, such as growth hormone and oestrogen. These hormonal changes differ between boys and girls. For instance, the level of oestrogen is by far higher in girls than boys at puberty. ${ }^{4}$

The mean age at presentation $(9.31 \pm 4.405 \mathrm{SD})$ in the present study was significantly higher than that reported from some other countries. ${ }^{11}$ The youngest child in our series was 7 months old. The reason for this difference is not clear. It might be related to racial variations.

About half (48\%) of our admitted children with DKA had positive family history of diabetes, of whom $52.5 \%$ were first degree relatives. Salman and associates study in Riyadh among 110 diabetic children between 1985 and 1989, found positive first degree family history of diabetes in only $28 \%$, where siblings accounted for $26 \%$, fathers for only $2 \%$ and none in mothers. ${ }^{12}$ We can say that the impact of a positive history of diabetes among siblings is stronger than due to other family members.

In the present study, $36 \%$ patient had severe form of DKA. This is in agreement with the frequency of severe DKA reported from Kuwait. ${ }^{13}$ On the other hand, the frequency of severe DKA observed in the present study is four-to-five fold higher than that reported from Finland. ${ }^{10}$ The reason for this difference might be that the background higher prevalence of DM in Finland makes the recognition of the disease easier by both the public and the physician, reducing delays in diagnosis and treatment, ultimately preventing severe form of DKA. The older age of our patients might be contributory, given that the frequency of severe form of DKA has been shown to be higher in patients between the ages 10-14 years compared to patients aged 5-9 years.

Consistent with the report of Habib, a major portion of the patients with impaired consciousness (total 46\% semiconscious and unconscious) at presentation in the present study, had severe form of DKA. In that study it was revealed that there was a strong correlation between degree of acidosis and depression of the central nervous system. ${ }^{14}$

When compared with reports from developed countries of the west, ${ }^{4}$ data from the present study revealed some differences in clinical characteristics of the patients.
For instance, the prevalence of DKA at first presentation of type 1DM, the mean age at presentation, the mean duration of symptoms before presentation and the mean blood glucose at the point of admission were all higher in the present series, representing some noteworthy features of diabetes in children and adolescents in a developing country. Similarly, reports from the Gulf countries such as Saudi Arabia, Kuwait, United Arab Emirates and Iran are generally in agreement with the findings in the present study. ${ }^{15,16}$. The implication is that the clinicians should be alert to these differences depending on where they are practicing.

The commonest precipitating factor in our study was infections. Worldwide, infection is the most common precipitating factor for DKA in both children and adults (30 - 50\%), mainly pneumonia and urinary tract infection. ${ }^{16}$ The study in North India between 1993 and 2000, among children with DKA showed that precipitating factors were sepsis (37\%), omitting insulin $(15 \%)$ and sepsis with insulin omission in $7 \% .{ }^{17} \mathrm{In}$ our study, omission of insulin contributed to only $10 \%$.

Among 35 (70\%) of children, DKA was the first clinical presentation of the disease. This is comparable to figures of $58 \%$ and $66 \%$ reported from India and Ethiopia respectively. ${ }^{17,18}$ In our study, polyuria and polydipsia were the commonest presenting symptom followed by weakness. This is similar to experiences from Saudi Arabia ${ }^{14}$ where vomiting and abdominal pain were the leading presenting symptoms in $98 \%$ of the cases; but is different from another study from India and Saudi Arabia where vomiting (71.3\%), and abdominal pain (66.3\%) were the commonest presenting symptoms. ${ }^{17,19}$ This difference is partly explained by the fact that most of our patients were in relatively elder age group. Thus polyuria and polydipsia were well appreciated.

All our patients were dehydrated with $52 \%$ had severe degree. This is unlike the experience from India where only $50 \%$ had clinical evidence of dehydration. ${ }^{17}$ It could well be that our patients arrived relatively late to the hospital or most of them had vomiting. In our study $46 \%$ of cases had altered level of consciousness with three $(6 \%)$ had being comatose. This is similar to a German experience where almost 23\% had altered level of consciousness, with $10.9 \%$ of them being comatose ${ }^{6}$; but is unlike the experience of another Saudi center 
where only $12.2 \%$ were said to had been drowsy with no cases of coma. ${ }^{14}$

No deaths occurred in our admitted children. The mortality rate from DKA in developed countries is from $0.15 \%$ to $0.31 \%$, with higher rate $(13 \%)$ being reported from developing countries where infections remains as one of the most important precipitating factors for DKA 5,17 . In a study done in Saudi Arabia no deaths occurred in children with DKA. ${ }^{14}$

Of the 50 cases studied, 18 (36\%) had severe acidosis, $16(32 \%)$ had moderate acidosis and $16(32 \%)$ had mild acidosis. Most patients had grossly elevated HbA1c, $41(82 \%)$ cases had HbA1c $>10 \%$ and $9(218 \%)$ cases had $\mathrm{HbA} 1 \mathrm{c}=7-10 \%$, and only 2 patient had $\mathrm{HbA} 1 \mathrm{c}$ $<8 \%$. In a study in Nairobi ${ }^{20},>90 \%$ patients had $\mathrm{HbA} 1 \mathrm{c}$ $>8 \%$.

In this study, most patients with mild to moderate acidosis did not have gross electrolyte imbalance. Most had normal or slightly low $\mathrm{Na}+$ levels, high normal or slightly elevated $\mathrm{K}+$ levels. Similar results were found in a study in Pakistan. ${ }^{21}$ Three patients had severe hyponatraemia and 4 patients had severe hypokalaemia. After initiation of treatment with fluid and insulin, almost all patients developed hypokalaemia requiring intravenous correction. In a national survey of Denmark 22 , similar observation appeared.

Most patients had grossly elevated blood sugar levels and ++ or more ketonuria on urine ketostix test. Patients were found to have ketonuria for longer period in comparison with their clinical and biochemical improvement evidenced by $\mathrm{pH}$ and $\mathrm{HCO}^{-}$levels. In the Indian study similar observation was reported ${ }^{23}$

Acute kidney injury was the leading complication encountered during the management of DKA in this series. It occurred in one-seventh of the cases, indicating the need for clinicians to be alert to its occurrence with the aim of preventing it due to DKA in this study.

\section{Limitation}

One limitation of the present study need to be considered that is the relatively small sample size from one healthcare facility. Future multicenter study with a larger sample size is being designed to enhance the interpretation of results, and ultimately strengthen conclusions. Despite this limitation, the study gave an insight into the clinical characteristics of newly- diagnosed diabetes presenting with DKA in our local community.

\section{Conclusion}

In this study infection was the commonest precipitating factor of DKA. Kussmaul's breathing and dehydration were the commonest clinical features. Most of the patients had improved after treatment. The glycaemic control in these patients was poor. Severe acidosis was common. In mild to moderate acidosis, gross electrolyte disturbances were infrequent but hypokalaemia developed after initiation of treatment. There was no death.

Conflict of interest: Nothing to declare.

\section{Disclaimer}

Research grant of taka 50000 was awarded for this study by Research Cell of BIRDEM General Hospital.

\section{References}

1. American Diabetes Association. Standards of Medical Care in Diabetes 2017 VIII: diabetes care in specific settings. Practice Guideline 2017.

2. World Health Organization. Global reports on Diabetes Mellitus: Report of a WHO/IDF Consultation. Geneva, Switzerland: World Health Organization 2016.

3. Norris AW, Wolfsdorf JI. Diabetes mellitus. In: Brook CGD, Clayton PE, Brown RS, eds. Brooks Clinical Pediatric Endocrinology. Oxford: Blackwell Publishing Ltd; 2005: 436473.

4. Wolfsdort J, Glaser N, Sperling MA. Diabetic ketoacidosios in infants, children and adolescents: a concensus statement from the American diabetes association. Diabetic Care 2006, 29(5):1150-59.

5. Cutis JR, To T, Muirhead S, Cumming E, Daneman D. Recent trends in hospitalization for diabetic ketoacidosis in Ontario children. Diabetic Care 2002; 25:1591-96.

6. Abdullah MA. Epidemiology of type 1 diabetes mellitus among Arab children. Saudi Med J 1994; 15 (4): 295-97.

7. Zabeen B, Nahar J, Mohsin F, Azad K, Nahar N. Diabetic ketoacidosis in children-an experience in a tertiary hospital. Ibrahim Med Coll J 2008; 2(1):17-20.

8. Rodacki M, Pereira JR, Nabuco de Oliveira AM, Barone B, MacDowell R. Ethnicity and young age influence the frequency of diabetic ketoacidosis at the onset of type 1 diabetes. Diabetes Res Clin Pract 2007; 78: 259-62.

9. Akanji AO. Clinical experience with adolescent diabetes in a Nigerian teaching hospital. Natl Med Assoc 1996, 88: $101-105$. 
10. Hekkala A, Knip M, Veijola R. Ketoacidosis at diagnosis of type 1 diabetes in children in Northern Finland: temporary changes over 20 years. Diabetes Care 2007; 30(4):861-66.

11. Mallare JT, Cordice CC, Ryan BA, Carey DE, Kreitzer PM, Frank GR. Identifying risk factors for development of diabetic ketoacidosis in new onset type 1 diabetes mellitus. Clin Pediatr (Phila) 2003; 42(7):591-97.

12. Salman H, Abanamy A, Hassan B, Khalil M. Childhood diabetes in Saudi Arabia. Diabet Med 1991; 8(2): 176-78.

13. Abdul RM, Al-Mahdi M, Al-Quttan H, Al-Tarkait N, Alhkouly M, Al-Safi R. Ketoacidosis at presentation of type 1 diabetes in children in Kuwait: frequency and clinical characteristics. Pediatr Diabetes 2010; 11(5):351-56.

14. Habib HS. Frequency and clinical characteristics of ketoacidosis as onset of childhood type 1 diabetes mellitus in Northwest Saudi Arabia. Saudi Med J 2005; 26(12):193639.

15. Punnose J, Agarwal MM, El Khadir A, Devadas K, Mugamer IT. Childhood and adolescent diabetes mellitus in Arabs residing in the United Arab Emirates. Diabetes Res Clin Pract 2002; 55(1):29-33.

16. Umpierrez GE, Latif K, Stoever J, Cuervo R, Park L, Freire AX et al. Efficacy of subcutaneous insulin lispro versus continuous intravenous regular insulin for the treatment of patients with diabetic ketoacidosis. Am J Med 2004; 117: 291-96.

17. Jayashree M, Singhi S. Diabetic ketoacidosis predictors of outcome in a pediatric intensive care unit of a developing country. Ped Cri Care Med 2004; 5(5): 427-33.

18. Della MT, Steinmetz L, Campos PR, Farhat SC, Schvartsman $\mathrm{C}$, Kuperman $\mathrm{H}$ et al. Subcutaneous use of a fast-acting insulin analog: an alternative treatment for pediatric patients with diabetic ketoacidosis. DiabetesCare 2005; 28:1856-61.

19. Qari F A. Precipitating Factors for Diabetic Ketoacidosis. Saudi Med J 2002; 23(2) : 173-76.

20. World Health Organization. Geneva, Switzerland: Definition and Diagnosis of Diabetes Mellitus and Intermediate Hyperglycaemia. 2006.

21. Kitabchi AE, Umpierrez GE, Murphy MB, Kreisberg RA. Hyperglycemic crises in adult patients with diabetes: a consensus statement from the American Diabetes Association. Diabetes Care 2006; 29: 2739-48.

22. Efstathiou SP, Tsiakou AG, Tsioulos DI, Zacharos ID, Mastorantonakis SE, Panagiotou TN, et al. A mortality prediction model in diabetic ketoacidosis. Clin Endocrinol 2002;57:595-601.

23. Joseph W, Nicole G, Mark AS. Diabetic ketoacidosis in infants, Children and Adolescents. Diabetes Care 2006; 29:1150-59. 